\title{
IMPROVEMENT IN RADIATION HARDNESS OF OXIDE BY SUCCESSIVE IRRADIATION-THEN-ANNEAL TREATMENTS
}

\author{
JENN-Gwo Hwu and SHYh-Liang Fu \\ Department of Electrical Engineering, National Taiwan University, Taipei, Taiwan, Republic of China
}

(Received 28 July 1988; in revised form 8 February 1989)

\begin{abstract}
It was experimentally observed that MOS devices prepared by repeated irradiation-then-anneal treatments were more radiation hard than those without such treatments. A modified isochronal annealing method based on the newly reported flat-band-condition annealing approximation was used in this work to examine the activation energy distribution of the radiation-induced positive charges. It was found that an MOS device after X-ray irradiation with a dose rate of $200 \mathrm{k}$ rads $\left(\mathrm{SiO}_{2}\right) / \min$ for $5 \mathrm{~min}$ exhibited a distribution of activation energy ranging from 1.2 to $1.8 \mathrm{eV}$. A sample which was subjected to a repeated irradiation-then-anneal treatments exhibited a significant reduction in the distribution of activation energy in the low energy region of $1.2-1.5 \mathrm{eV}$, but not in the high energy region of $1.5-1.8 \mathrm{eV}$. The activation energy distribution for samples prepared under various postoxidation treatments are discussed.
\end{abstract}

\section{INTRODUCTION}

A radiation-hard oxide is important for an integrated-circuit (IC) to achieve long-term stability. Several methods are proposed to make the oxides more radiation-hard[1-3]. These workers employed fabrication variations, e.g. process optimization[1] or double layer structure[3], to attain their goals. It is essential that the fabrication procedures used to obtain the radiation-hard oxides should be compatible with the existing IC processes.

In this work, we propose a simple way to efficiently reduce the radiation sensitivity of oxide which requires no fabrication variations. It is well known that the number of radiation-induced positive charges near the $\mathrm{SiO}_{2} / \mathrm{Si}$ interface is strongly dependent on the interfacial strain[4-6]. The larger the interfacial strain, the worse the radiation hardness of oxide. It can be explained by a model in which the ionizing radiation breaks a strained bond leaving an $\mathrm{Si}^{+}$site, which is the trapped hole, and a mobile nonbridging oxygen that propagates, by the action of the strain gradient, toward the interface[4]. Therefore, we believe that radiation releases the interfacial strain although it introduces positive charges near the $\mathrm{SiO}_{2} / \mathrm{Si}$ interface. Fortunately, most of the radiationinduced positive charges can be annealed out at a suitable temperature or ambient[7,8]. So, if a sample is first irradiated and then annealed, it will become more radiation hard than that without such treatment since its interfacial strain has been suitably released. This phenomenon was experimentally observed in this work based on the $C-V$ measurements. The irradiation-then-anneal procedure can be repeatedly carried out until the sample reaches a certain saturated radiation hardness level, beyond which there is no significant improvement.
In addition to the $C-V$ measurements, the activation energy distributions for the samples with and without successive irradiation-then-anneal treatments were also examined. There are several ways to obtain the activation energy distributions of the radiation induced damage[7,9-11]. We realized that the residual charges left behind the metal gate (or the substrate) of an MOS device after $C-V$ measurement might affect the anneal behavior significantly. A technique called flat-band-condition annealing approximation was proposed to exclude the fieldassisted effect during annealing[12]. In this work, a modified isochronal annealing method was developed based on the above technique. The activation-energy distribution of an X-ray irradiated sample was found to be in the range of $1.2-1.8 \mathrm{eV}$. It was observed that the change of effective charge number density in the low activation energy range of $1.2-1.5 \mathrm{eV}$ was reduced by successive irradiation-then-anneal treatments or by postoxidation cooling in an ambient containing oxygen, but no such reduction in the change of effective charge number density was seen in the high activation energy range of $1.5-1.8 \mathrm{eV}$. We give some possible explanations for this phenomenon based on the strain-related mechanism.

\section{EXPERIMENTAL}

$p$-Type and $n$-type silicon wafers with (100)orientation were used as the substrates of the MOS devices. After standard cleaning procedures, these wafers were dry oxidized at $900^{\circ} \mathrm{C}$ in a $(3 \%) \mathrm{HCl}$ ambient. After oxidization, most of the samples were first postoxidation annealed (P.O.A.) and then cooled in an $\mathrm{N}_{2}$ ambient. It is known that samples prepared under various oxidation conditions will exhibit 
different sensitivities to irradiation[1]. In order to compare the radiation hardness between the sample prepared by successive irradiation-then-anneal treatments and that by a radiation-hard oxidation process, some samples after oxidization were cooled in an $\mathrm{N}_{2}+\mathrm{O}_{2}$ ambient without P.O.A. treatment. The final oxide thickness of these samples was about $350 \AA$. Aluminum was used as the material for the gate and the backside contacts. Finally, the MOS devices were postmetallization annealed at $400^{\circ} \mathrm{C}$ in an $\mathrm{N}_{2}$ ambient for about $30 \mathrm{~min}$.

An $\operatorname{MOS}(p)$ device (P.O.A. and cooled in $\mathrm{N}_{2}$ ), i.e. sample $A$, was successively irradiated and then annealed. A dose rate of $200 \mathrm{k}$ rads $\left(\mathrm{SiO}_{2}\right) / \mathrm{min}$ and an irradiation time of $5 \mathrm{~min}$ were used for $\mathrm{X}$-ray irradiation. An anneal temperature of $400^{\circ} \mathrm{C}$ and an anneal time of $30 \mathrm{~min}$ were employed. After first anneal, the capacitance values were measured when the gate voltage was varied from -4 to $+2 \mathrm{~V}$ and then from +2 to $-4 \mathrm{~V}$. Such a characteristic is shown as curve no. 1 in Fig. 1(a). The device was subjected to irradiation and the above $C-V$ measurement was repeated. The $C-V$ characteristics are shown by two curves marked "1" and the two curves are due to the hysteresis loop. The hysteresis is due to increased slow trap states. The successive annealing resulted in curve 2 and the subsequent irradiation resulted in curves 2 '. The $C-V$ characteristics after the fourth annealing and irradiation are shown by curves 3 and 3 'respectively.

The flat-band voltage $V_{\mathrm{FB}}$ obtained from the $C-V$ characteristics are shown in Fig. 1(b). The plot of $V_{\mathrm{FB}}$ as a function of number of anneal/irradiation treatments is shown in the figure. There are two values of $V_{\mathrm{FB}}$ for an irradiation due to the hysteresis loop. The value of $V_{\mathrm{FB}}$ remained practically constant after each annealing treatment. That is to say, the radiation- induced damage can be removed by annealing. Interestingly, the radiation sensitivity of this device decreases, i.e. the value of $V_{\mathrm{FB}}$ after $\mathrm{X}$-ray irradiation and subsequent anneal becomes small, as the number of successive irradiation-then-anneal treatment increases. After about four such treatments, the radiation sensitivity becomes almost saturated. Under such circumstances, the flat-band voltage shift $\Delta V_{\mathrm{FB}}$ after X-ray irradiation is reduced to about the half of that without treatment. The radiation hardness of the oxide had finally been significantly improved.

Similarly, an $\operatorname{MOS}(n)$ device (P.O.A. and cooled in $\mathrm{N}_{2}$ ), i.e. sample $\mathrm{B}$, with an oxide thickness of $350 \AA$ was also examined. Figure 2(a) shows some of the obtained $C-V$ curves. It is noted that the radiation behavior of an $\operatorname{MOS}(n)$ device is generally different from that of an $\operatorname{MOS}(p)$ device[13,14]. Briefly, the variation of the $V_{\mathrm{FB}}$ for an $\operatorname{MOS}(n)$ device after irradiation is much smaller than that for an $\operatorname{MOS}(p)$ device. The effects of work function difference[13] and interface states[14] mainly account for this phenomenon. In order to describe the radiation hardness property clearly, only two sets of $C-V$ curves are presented in this figure. Curve no. 1 is the $C-V$ curve after the first annealing. After the first irradiation, the $C-V$ curve is seriously distorted, as shown by curve no. 1'. The irradiation-then-anneal treatment was repeatedly carried out as described above. Curve no. 2 is the $C-V$ curve after the seventh annealing, which coincides with curve no. 1. After the seventh irradiation, the $C-V$ curve is still distorted, as shown by curve no. $2^{\prime}$. However, it is obvious that this curve is steeper than curve no. $1^{\prime}$. This is due to the fact that the number of interface states for curve no. $2^{\prime}$ is much smaller than that for curve no. 1'. Also, when we compare the mid-gap shifts for these curves, we see that the number of the net fixed charges for

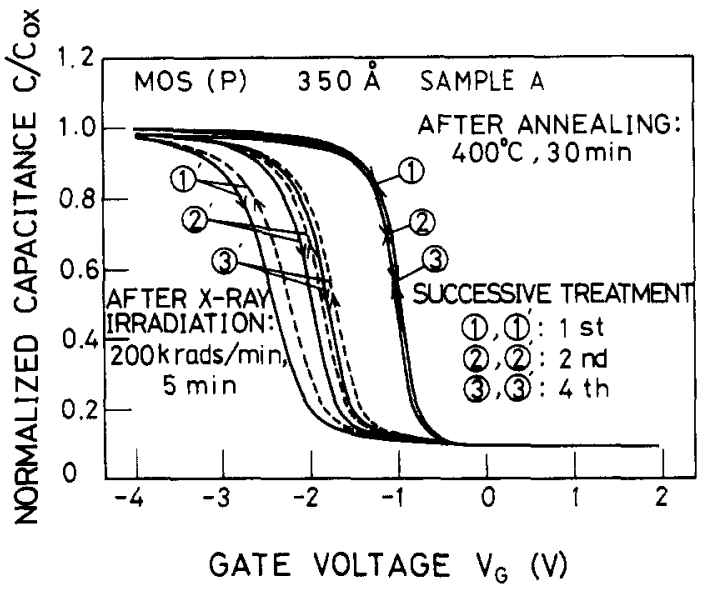

(a)

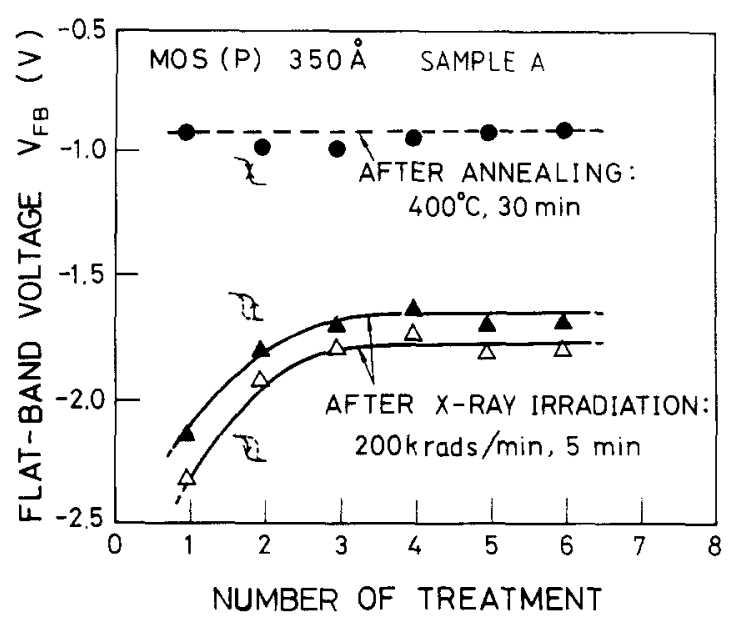

(b)

Fig. 1. (a) The $C-V$ curves of an $\operatorname{MOS}(p)$ device after the first, the second, and the fourth annealings and irradiations. (b) The flat-band voltage $V_{F B}$ vs the number of treatment for this device after each annealing or irradiation. 


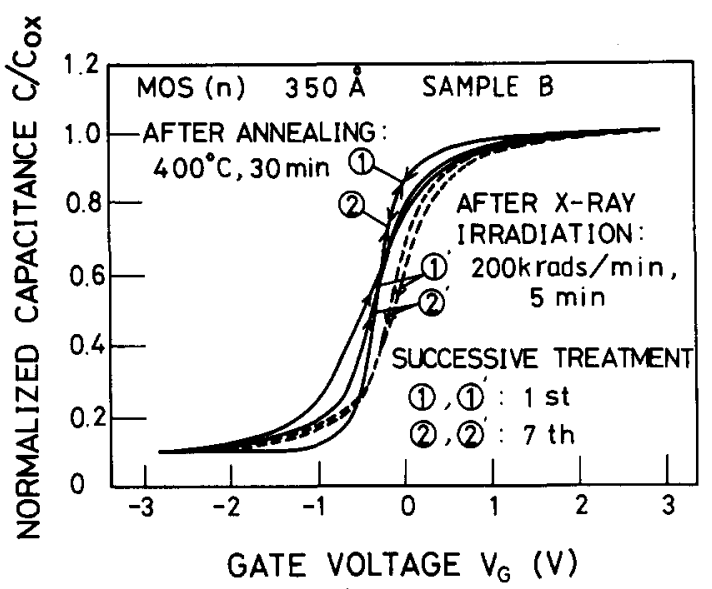

(a)

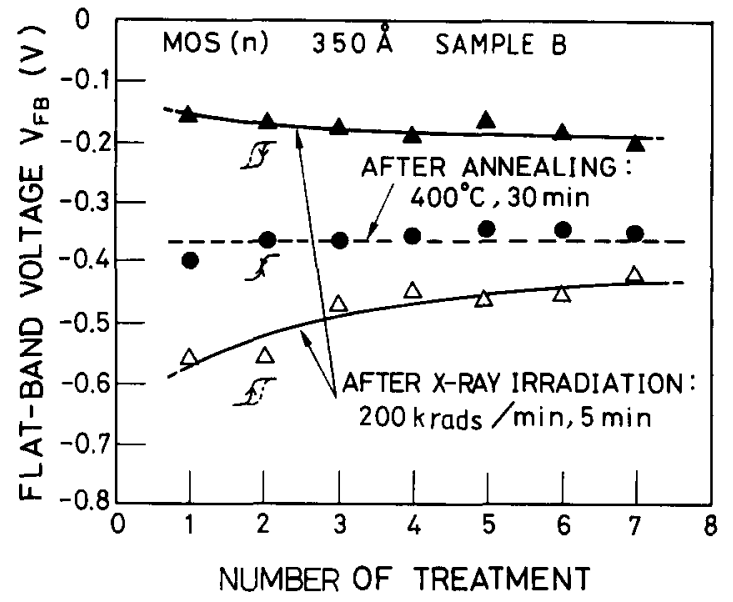

(b)

Fig. 2. (a) The $C-V$ curves of an $\operatorname{MOS}(n)$ device after the first and the seventh annealings and irradiations. (b) The flat-band voltage $V_{\mathrm{FB}}$ vs the number of treatment for this device after each annealing or irradiation.

curve no. $2^{\prime}$ is smaller than that for curve no. $1^{\prime}$. Figure 2(b) shows $V_{\mathrm{FB}}$ vs the number of treatment of this device after each annealing or irradiation. It is also clear from these figures that the radiation hardness of the oxide had been improved by the successive irradiation-then-anneal treatments.

In order to show the importance of carrying out the irradiation steps in the hardening procedure, i.e. the successive irradiation-then-anneal treatments, some other samples (P.O.A. and cooled in $\mathrm{N}_{2}$ ) were examined as follows. Four $\operatorname{MOS}(p)$ and four $\operatorname{MOS}(n)$ devices with oxide thickness of $350 \AA$ were postmetallization annealed in an $\mathrm{N}_{2}$ ambient for $0.5,1,2$ and $3 \mathrm{~h}$, respectively. Then, they were $\mathrm{X}$-ray irradiated with the same condition as those mentioned in Figs 1 and 2. Figures 3(a) and (b) show $V_{\mathrm{FB}}$ vs postmetallization anneal time for the $\operatorname{MOS}(p)$ and the $\operatorname{MOS}(n)$ devices, respectively, before and after irradiations. No improvement of radiation hardness is observed when postmetallization anneal time is increased. The irradiation-then-anneal procedure improves the radiation. The radiation hardness is not achievable by merely increasing the anneal time without the irradiation step in between. Therefore the theory that the radiation releases the interfacial strain, and the subsequent annealing removes the positive charges introduced during irradiation, seems valid. For the samples prepared by a radiation-hard oxidation process, the irradiation experiments were also carried out. In Fig. 4, two $\operatorname{MOS}(n)$ devices, i.e. samples C and $\mathrm{D}$, and two $\operatorname{MOS}(p)$ devices, i.e. samples $\mathrm{E}$ and $F$, are compared. Samples $C$ and $E$ were prepared by a regular process (P.O.A. and cooled in $\mathrm{N}_{2}$ ), while samples $D$ and $F$ by a radiation-hard oxidation process (no P.O.A., cooled in $\mathrm{N}_{2}+\mathrm{O}_{2}$ ). Figure 4(a) shows the $C-V$ curves of samples $\mathrm{C}$ and $\mathrm{D}$ before and after X-ray irradiations. Curves no. 1 and $1^{\prime}$ are the $C-V$ curves of sample $\mathrm{C}$ before and after irradiation, respectively, while curves no. 2 and $2^{\prime}$ are the $C-V$ curves of sample $\mathrm{D}$ before and after irradiation,

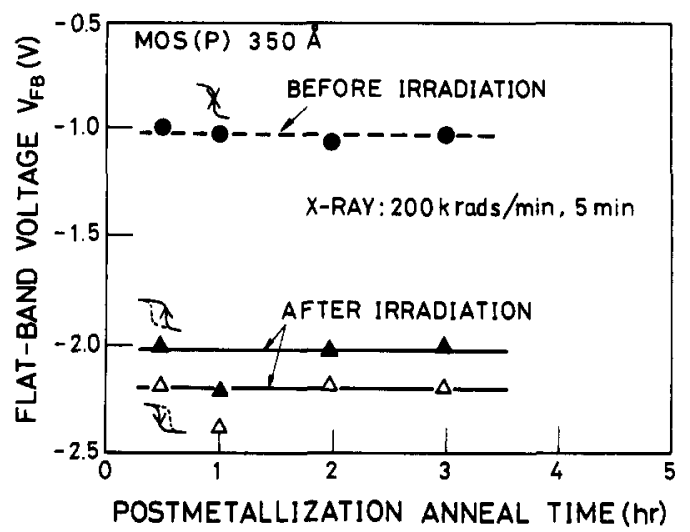

(a)

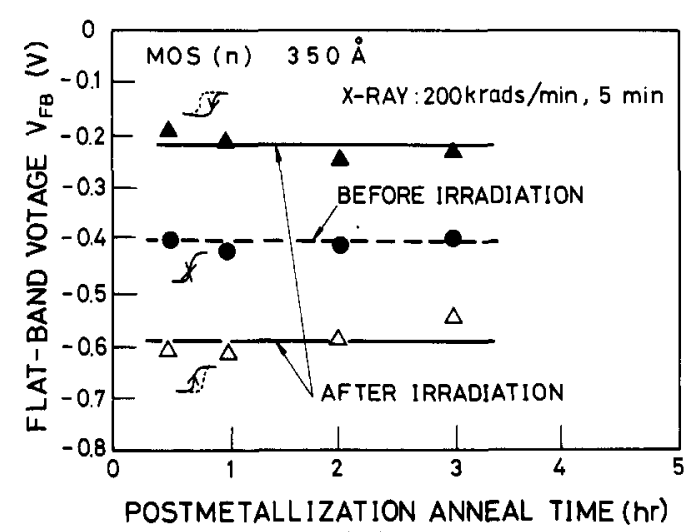

(b)

Fig. 3. The flat-band voltage $V_{\mathrm{FB}}$ vs the postmetallization anneal time for (a) an $\operatorname{MOS}(p)$ and (b) $\operatorname{MOS}(n)$ devices before and after irradiations. 
respectively. As can be seen from this figure, before irradiation, sample D (i.e. curve no. 2) contains more positive fixed charges than sample $C$ (i.e. curve no. 1). This phenomenon probably accounts for the excess oxygen left at the $\mathrm{SiO}_{2} / \mathrm{Si}$ interfacial region during the cooling procedure in an ambient containing oxygen. However, after irradiation, sample D (i.e. curve no. $\left.2^{\prime}\right)$ is less sensitive to irradiation than sample $\mathrm{C}$ (i.e. curve no. $1^{\prime}$ ). Similarly, $\operatorname{MOS}(p)$ devices also appear to have the same radiation hardness as observed above. In Fig. 4(b), curves no. 3 and $3^{\prime}$ are the $C-V$ curves of sample $E$ before and after irradiation, respectively, while curves no. 4 and $4^{\prime}$ are the $C-V$ curves of sample $\mathrm{F}$ before and after irradiation, respectively. Before irradiation, curve no, 4 contains more positive fixed charges than curve no. 3. After irradiation, curve no. $4^{\prime}$ is less sensitive to irradiation than curve no. $3^{\prime}$. It is believed that the excess oxygen releases some of the interfacial strain and therefore hardens the oxide[15]. However, the release of interfacial strain due to excess oxygen is not so efficient as that by successive irradiation-then-anneal treatments. They will be compared in a later section.

\section{ANALYSIS OF ACTIVATION ENERGY DISTRIBUTION}

The change of effective charge number density as a function of activation energy is one of the important characteristics in examining the differences of the radiation effects between two different samples[9-11]. It is believed that the $C-V$ technique was usually used for the analysis. However, the final voltage of each $C-V$ measurement should be carefully considered since the residual charges left behind the metal gate (or the substrate) of an MOS device can exist for a long time even when the bias source is removed from the sample, and will cause varied annealing behavior due to the differences in the electric field $\left(E_{\mathrm{i}}\right)$ at the $\mathrm{SiO}_{2} / \mathrm{Si}$ interface during annealing[12]. Because the characteristics of the existing residual charges at a particular annealing temperature is important to the analysis of the modified isochronal annealing method proposed later in this work, a more detailed characterization of the behavior of the existing residual charges at various temperatures should be carried out.

An experimental setup used for studying the decay characteristics of an MOS device as a function of temperature is shown in Fig. 5(a). The sample under test was charged to $-5 \mathrm{~V}$ just before the start of the experiment. The decay behavior of the floating gate voltage $V_{G}$ was measured with a high input impedance electrometer (Keithley $602 \mathrm{C}$ ). Figure 5(b) shows the results of an $\operatorname{MOS}(p)$ device tested at 27 , $200,250,300,325,350$ and $375^{\circ} \mathrm{C}$, respectively. The corresponding RC time constants, which were determined according to the $V_{\mathrm{G}}$ 's read at $t=5 \mathrm{~min}$, are also shown in this figure. It is clear from this figure that the decay of $V_{\mathrm{G}}$ at $t=2 \mathrm{~min}$, which was chosen

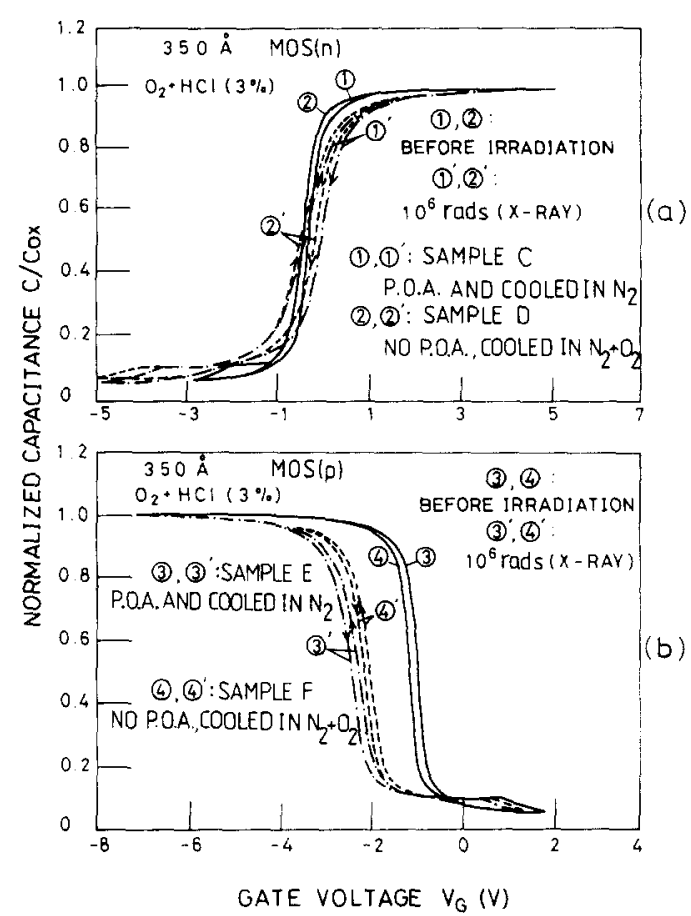

Fig. 4. (a) The $C-V$ curves of two $\operatorname{MOS}(n)$ devices before and after irradiations. Sample $C$ is prepared by a regular oxidation process (P.O.A. and cooled in $\mathrm{N}_{2}$ ), while sample $D$ by a radiation-hard oxidation process (no P.O.A. cooled in $\mathrm{N}_{2}+\mathrm{O}_{2}$ ). (b) The $C-V$ curves of two $\operatorname{MOS}(p)$ devices before and after irradiations. Sample $E$ is prepared by a regular oxidation process (P.O.A., and cooled in $\mathrm{N}_{2}$ ), while sample $F$ by a radiation-hard oxidation process (no P.O.A., cooled in $\mathrm{N}_{2}+\mathrm{O}_{2}$ ).
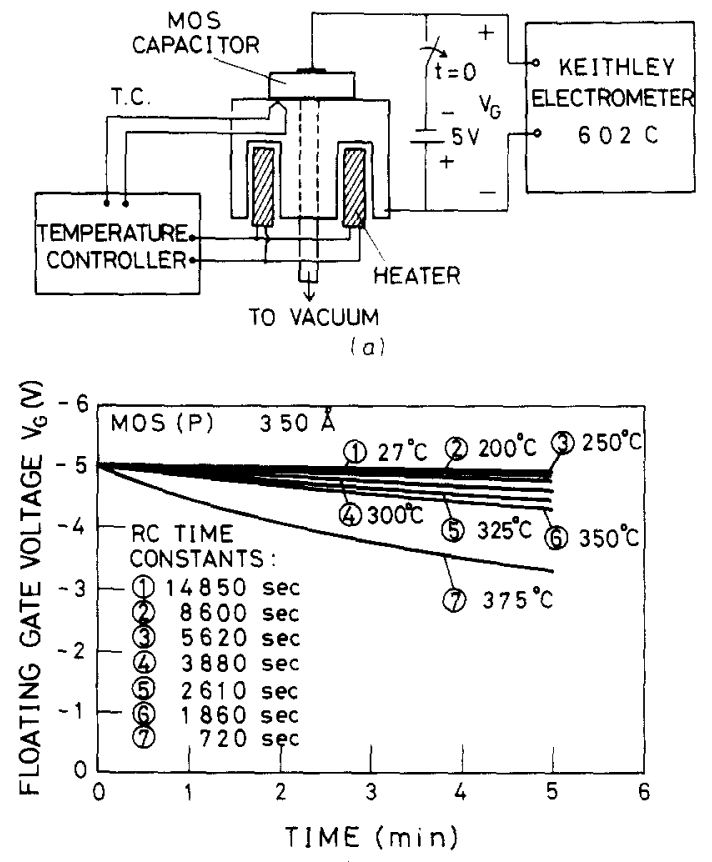

(b)

Fig. 5. (a) An experimental setup used for studying the decay characteristics of an MOS device as a function of temperature. (b) The decay behavior of an $\operatorname{MOS}(p)$ device tested at $27,200,250,300,325,350$ and $375^{\circ} \mathrm{C}$, respectively, from $-5 \mathrm{~V}$ 
as the anneal time in each isochronal anneal as discussed later, is very small below $350^{\circ} \mathrm{C}$.

For an MOS device with a flat-band voltage of $V_{\mathrm{FB}}$, the relationship between the gate voltage $V_{\mathrm{G}}$ and the $E_{\mathrm{i}}$ can be expressed as:

$$
V_{\mathrm{G}}=V_{\mathrm{FB}}+E_{\mathrm{i}} d_{\mathrm{ox}}+\psi_{\mathrm{s}},
$$

where $d_{\mathrm{ox}}$ is the oxide thickness and $\psi_{\mathrm{s}}$ is the semiconductor surface potential. In order to maintain identical conditions during the annealing procedure, it was decided to keep $E_{\mathrm{i}}=0 \mathrm{~V} / \mathrm{cm}$. Then it was necessary to charge the device to $V_{\mathrm{FB}}^{\prime}$, and $V_{\mathrm{FB}}^{\prime}$ was the flat-band voltage measured by a $C-V$ measurement before each flat-band-condition anneal. It is noted that if an irradiated MOS device is tested for $C-V$ characteristics with a constant sweep rate in both the forward and the backward directions, two $V_{\mathrm{FB}}$ 's (see Figs 1 and 2) are obtained due to the existence of slow trap states. In order to exclude the effect of slow trap states on $V_{\mathrm{FB}}$, the d.c. bias was maintained at the flat-band condition for a sufficiently long time. By placing this charged device in an annealing furnace with a temperature below $350^{\circ} \mathrm{C}$ for about $2 \mathrm{~min}$, the flat-band-condition annealing approximation is achieved. Figure 6 shows the change of effective charge number density $\Delta N_{\text {eff }}$ vs time for two Co-60 irradiated $\operatorname{MOS}(p)$ devices annealed at $200^{\circ} \mathrm{C}$ under the flat-band condition and the short-circuit condition, i.e. $V_{\mathrm{G}}=0$. The $\Delta N_{\text {eff }}$ was obtained from the flat-band voltage shift $\Delta V_{\mathrm{FB}}$ (with respect to the initial $V_{\mathrm{FB}}$ ) according to the following equation:

$$
\Delta N_{\mathrm{eff}}=\frac{C_{\mathrm{ox}}}{q} \Delta V_{\mathrm{FB}},
$$

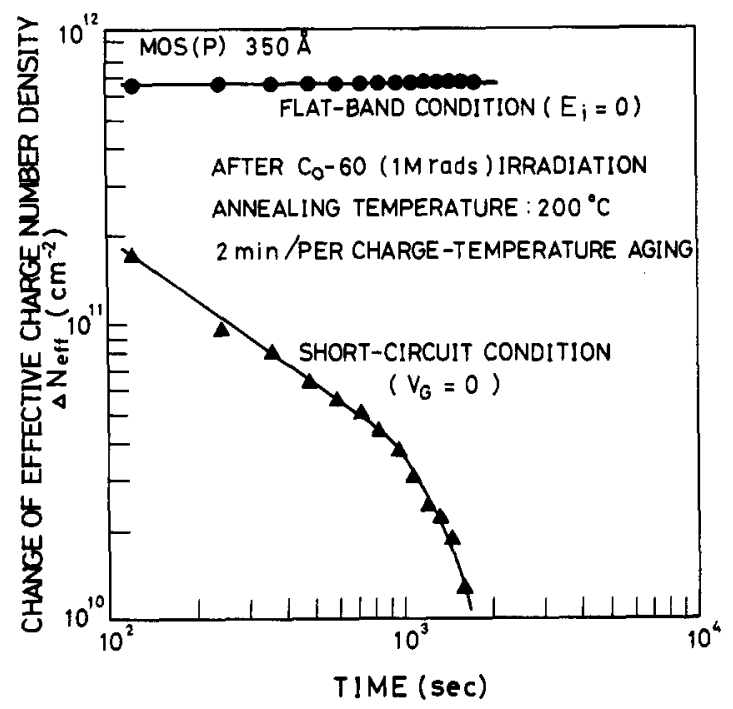

Fig. 6. The change of effective charge number density $\Delta N_{\text {eff }}$ vs time for two Co-60 irradiated $\operatorname{MOS}(p)$ devices annealed at $200^{\circ} \mathrm{C}$ under the flat-band and the short-circuit conditions, respectively.

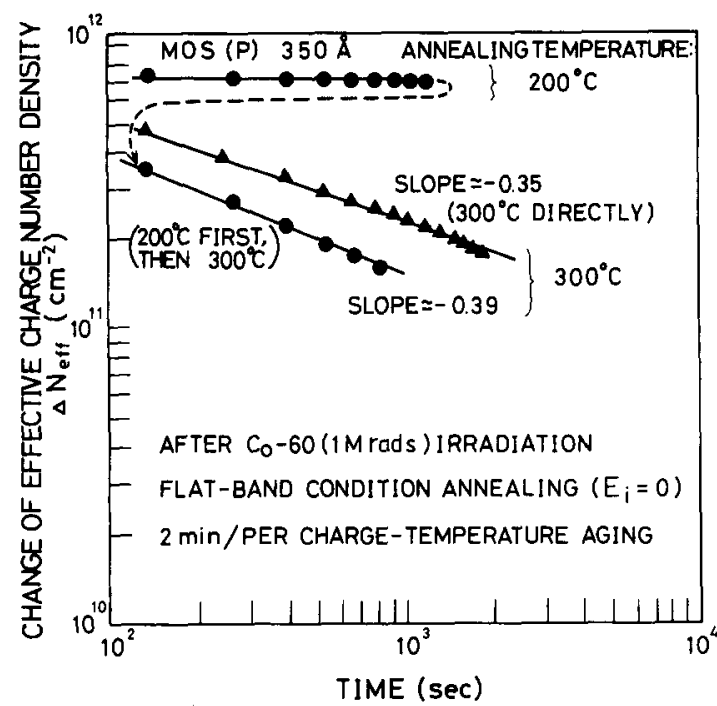

Fig. 7. The change of effective charge number density $\Delta N_{\text {eff }}$ vs time for two $\operatorname{MOS}(p)$ devices annealed under the flatband conditions. The solid circles are the results of a sample first annealed at $200^{\circ} \mathrm{C}$ then subsequently at $300^{\circ} \mathrm{C}$ while the solid triangles are the results of another sample annealed at $300^{\circ} \mathrm{C}$ only.

where $C_{\mathrm{ox}}$ is the oxide capacitance. It is interesting to note that the $\Delta N_{\text {eff }}$ obtained under the flat-band condition varies slowly with time, while the $\Delta N_{\text {eff }}$ obtained under the short-circuit condition changes rapidly with time. The $E_{\mathrm{i}}$ is positive under the shortcircuit condition. Thus the annealing behavior is affected due to the detrapping of the holes from $\mathrm{SiO}_{2}$ into $\mathrm{Si}[16]$. The $E_{\mathrm{i}}$ thus plays an important role in the annealing behavior.

Temperature is also an important factor in determining the annealing behavior. Figure 7 shows $\Delta N_{\text {eff }}$ vs time for two $\operatorname{MOS}(p)$ devices annealed under the flat-band conditions. The ones denoted by the solid circles were obtained from a sample annealed first at $200^{\circ} \mathrm{C}$ then subsequently at $300^{\circ} \mathrm{C}$, while the ones denoted by the solid triangles were obtained from a second sample annealed at $300^{\circ} \mathrm{C}$ only. For the former ones annealed at $200^{\circ} \mathrm{C}$, the $\Delta N_{\text {eff }}$ changes very slowly with time, as observed in Fig. 6 . As the temperature is increased to $300^{\circ} \mathrm{C}$ the $\Delta N_{\text {eff }}$ decreases linearly with time in a log plot. The observation is the same for the second case where the sample was annealed at $300^{\circ} \mathrm{C}$ only. The slope for the first case is -0.39 . While for the later one the slope is -0.35 . It is noted that a linear relationship between $\Delta N_{\text {eff }}$ and time in log scale implies a power law dependence of $\Delta N_{\text {eff }}$ on time[17]. The slope mentioned above represents the exponent of time, which is unitless in such case. Although the two reaction model proposed by Reed and Plummer[17] can partially explain the linear slope. The explanation for the temperature dependence of the slope is still lacking. The temperature dependence of the annealing behavior of $\Delta N_{\text {eff }}$ is examined by another method, the activation energy distribution. 
Generally, the activation energy $E_{a}$ for an annealing process carried out at a temperature $T$ for $t \mathrm{~s}$ can be approximately expressed by $[9,18,19]$ :

$$
E_{\mathrm{a}} \cong k T \ln \left(v_{0} t\right) \text {. }
$$

where $v_{0}$ is a frequency factor. It is noted that the dependence of $E_{\mathrm{a}}$ on $v_{0}$ is very weak, so the specific value of $v_{0}$ is not critical $[1,19]$. In this work $v_{0}=10^{12} \mathrm{~s}^{-1}$ was used. The flat-band-condition approximation is valid below $350 \mathrm{C}$, and the annealing characteristic is constant below $200^{\circ} \mathrm{C}$ as observed in Figs 6 and 7, a modified isochronal annealing method is proposed as follows. The device was annealed at flat-band condition for $120 \mathrm{~s}$ at temperatures from $150^{\circ} \mathrm{C}$ to $350^{\circ} \mathrm{C}$ in $10 \mathrm{C}$ increments. Then, the $E_{\mathrm{a}}$ for each annealing step was calculated using eqn (3) with $t=120 \mathrm{~s}$ and $T$ as a parameter. It was found that the activation energies varied from 1.2 to $1.8 \mathrm{eV}$. Figure 8(a) shows the effective charge number density $N_{\text {eff }}$ vs $E_{\text {a }}$ for an X-ray irradiated $\operatorname{MOS}(p)$ device, i.e. sample $E$ annealed under the flat-band condition. It is noted that the $N_{\text {eff }}$ was obtained from the flat-band voltage shift with respect to the ideal $V_{\mathrm{FB}}\left(V_{\mathrm{FB}}=-0.88 \mathrm{~V}\right)$. The activation energy distribution can be calculated by taking the negative derivative of $N_{\text {eff }}$ with respect to $E_{\mathrm{a}}$, i.e. $-\mathrm{d} N_{\mathrm{etf}} / \mathrm{d} E_{\mathrm{a}}$, as shown in Fig. 8(b).

We compared the activation energy distributions of three $\operatorname{MOS}(p)$ devices, i.e. samples $\mathrm{A}, \mathrm{E}$ and $\mathrm{F}$, prepared in different ways. Sample A was prepared by successive irradiation-then-anneal treatments as described in Fig. 1, sample $E$ by a regular oxidation process (P.O.A. and cooled in $\mathrm{N}_{2}$ ), and sample $\mathrm{F}$ by a radiation-hard oxidation process (no P.O.A., cooled in $\mathrm{N}_{2}+\mathrm{O}_{2}$ ) as described in Fig. 4. Their activation energy distributions are shown in Fig. 9. It was observed that all the activation energies were
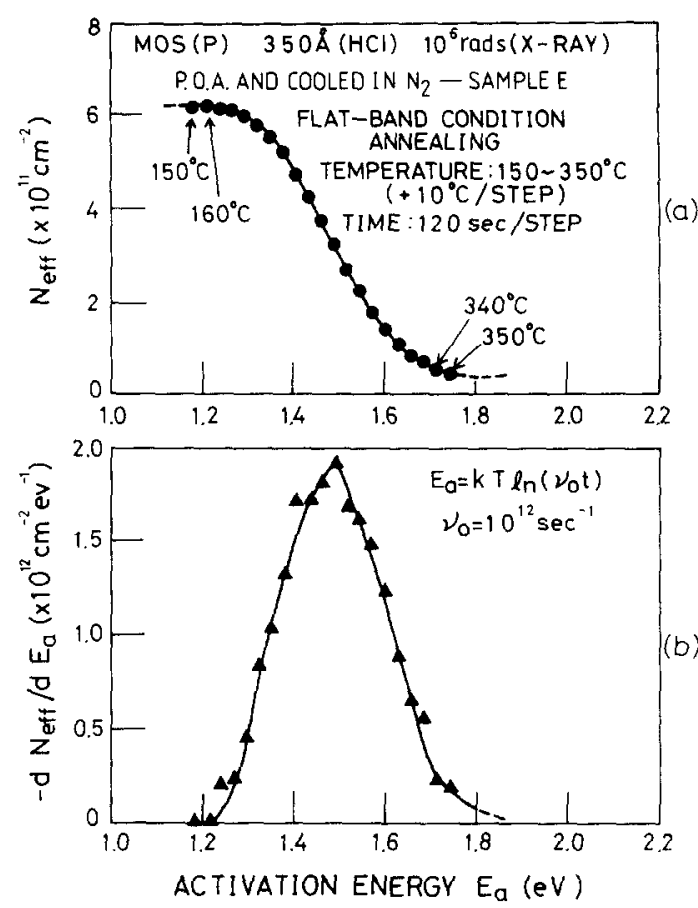

Fig. 8. (a) The effective charge number density $N_{\text {eff }}$ vs the activation energy $E_{\mathrm{a}}$ for an X-ray irradiated device annealed under the flat-band condition for $120 \mathrm{~s}$ at temperatures from $150^{\circ} \mathrm{C}$ to $350^{\circ} \mathrm{C}$ in $10^{\circ} \mathrm{C}$ increments. (b) The change of effective charge number density, $-\mathrm{d} N_{\text {eff }} / \mathrm{d} E_{\mathrm{a}}$, vs the activation energy $E_{\mathrm{a}}$ of the device mentioned in (a).

ranged from 1.2 to $1.8 \mathrm{eV}$, and the distributions of $\left(-\mathrm{d} N_{\mathrm{eff}} / \mathrm{d} E_{\mathrm{a}}\right)$ in the high energy region of $1.5-1.8 \mathrm{eV}$ for all samples are almost the same. However, near the low energy range of $1.2-1.5 \mathrm{eV}$, the distribution of $\left(-\mathrm{d} N_{\mathrm{eff}} / \mathrm{d} E_{\mathrm{a}}\right.$ ) for sample $\mathrm{A}$ is the smallest one among three, and that for sample $F$ is smaller than that for

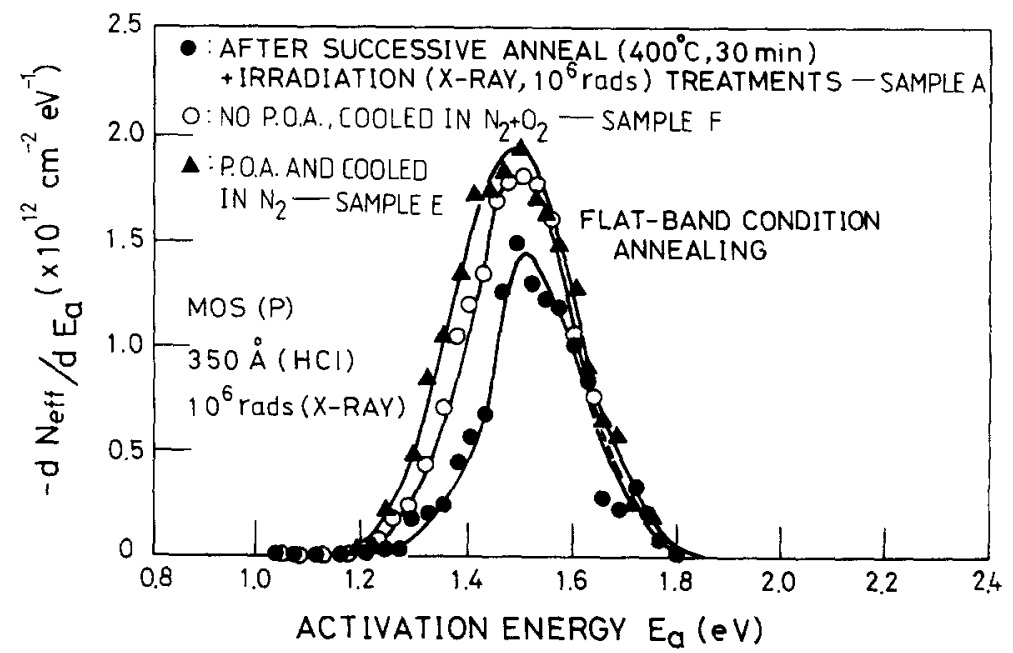

Fig. 9. $-\mathrm{d} N_{\mathrm{eff}} / \mathrm{d} E_{\mathrm{a}}$ vs the activation energy for three X-ray irradiated $\operatorname{MOS}(p)$ devices annealed under the flat-band conditions. Sample A was prepared by successive irradiation-then-anneal treatments, sample $E$ by a regular oxidation process (P.O.A. and cooled in $\mathrm{N}_{2}$ ) and sample $F$ by a radiation-hard oxidation process (no P.O.A., cooled in $\mathrm{N}_{2}+\mathrm{O}_{2}$ ). 
sample E. Therefore, a "hardening" procedure can reduce the distribution of $\left(-\mathrm{d} N_{\text {eff }} / \mathrm{d} E_{\mathrm{a}}\right)$ near the low energy region. Interestingly, the "hardening" procedure of successive irradiation-then-anneal treatments is more efficient in the reduction than that of changing oxidation condition only. This observation is different from the result obtained in the Shanfield work[20]. It is probably due to the fact that the effect of the hardening process used in this work, i.e. successive irradiation-then-anneal treatments, is basically different from the effect of that used in the Shanfield work, i.e. postoxidation annealing. From the behavior observed in Fig. 9, it is believed that the low activation energies are related to the straininduced hole trap centers, which can be eliminated by releasing the strain near the $\mathrm{SiO}_{2} / \mathrm{Si}$ interfacial region via the "hardening" procedure, while the high activation energies are related to the intrinsic hole trap centers and are not affected by the oxide treatment. This explains the saturation behavior of the radiation hardness level observed in Figs 1 and 2.

\section{CONCLUSIONS}

Successive irradiation-then-anneal treatments provide a possible way for one to improve the radiation hardness of oxide. The modified isochronal annealing method used in this work excludes the field-assisted effect on annealing. Based on this method, the activation energy for the irradiation-induced damage was found to be within the range of $1.2-1.8 \mathrm{eV}$. The change of effective charge number density in the low activation energy range of $1.2-1.5 \mathrm{eV}$ was reduced by the radiation hardening procedures proposed in this work and it is possibly due to the reduction in the strain-induced hole trap centers. But in the high activation energy range of $1.5-1.8 \mathrm{eV}$, such a reduction was not observed and it is possibly due to intrinsic hole trap centers.
Acknowledgement - The authors want to thank the National Science Council of the Republic of China for supporting this work under Contract No. NSC77-0404-E002-09.

\section{REFERENCES}

1. G. F. Derbenwick and B. L. Gregory, IEEE Trans. Nucl. Sci. NS-22, 2151 (1975).

2. G. J. Hu, J. M. Aitken and R. H. Dennard, IEEE Trans. Nucl. Sci. NS-28, 4102 (1981).

3. K. Kasama, F. Toyokawa, M. Sakamoto and K. Kobayashi, IEEE Trans. Nucl. Sci. NS-32, 3965 (1985)

4. F. J. Grunthaner, P. J. Grunthaner and J. Maserjian, IEEE Trans. Nucl. Sci. NS-29, 1462 (1982).

5. S. I. Raider and R. Flitsch, J. Electrochem. Soc. 123, 1754 (1976).

6. V. Zekeriya and T. P. Ma, Appl. Phys. Lett. 47, 54 (1985).

7. E. H. Snow, A. S. Grove and D. J. Fitzgerald, Proc. IEEE 55, 1168 (1967).

8. E. H. Nicollian and J. R. Brews, MOS (Metal Oxide Semiconductor) Physics and Technology, p. 798. WileyInterscience, New York (1982).

9. V. Danchenko, E. G. Stassinopoulos, P. H. Fang and S. S. Brashears, IEEE Trans. Nucl. Sci. NS-27, 1658 (1980).

10. D. B. Brown, D. I. Ma, C. M. Dozier and M. C. Peckerar, IEEE Trans. Nucl. Sci. NS-30, 4059 (1983).

11. Z. Shanfield, IEEE Trans. Nucl. Sci. NS-30, 4064 (1983).

12. J. G. Hwu. G. S. Lee, S. C. Lee and W. S. Wang, IEEE Trans. Nucl. Sci. NS-35, 960 (1988).

13. K. Suzuki, M. Sakagami, E. Nishimura and K. Watanabe, IEEE Trans. Nucl. Sci. NS-32, 3911 (1985).

14. G. A. Scoggan and T. P. Ma, J. appl. Phys. 48, 294 (1977).

15. J. G. Hwu and S. L. Fu, Appl. Phys. A. 46, 221 (1988).

16. S. K. Haywood and R. F. Dekeersmaecker, Appl. Phys. Lett. 47, 381 (1985).

17. M. L. Reed and J. D. Plummer, IEEE Trans. Nucl. Sci. NS-33, 1198 (1985).

18. W. Primak, Phys. Rev. 100, 1677 (1955).

19. M. Simons and H. L. Hughes, IEEE Trans. Nucl. Sci. NS-19, 282 (1972).

20. Z. Shanfield and M. M. Moriwaki, IEEE Trans. Nucl. Sci. NS-32, 3929 (1985). 